\title{
Polymer microfluidic devices: an overview of fabrication methods
}

\begin{abstract}
Raquel O. Rodrigues ${ }^{1}$, Rui Lima ${ }^{2}$, Helder T. Gomes ${ }^{3}$, Adrián M. T. Silva ${ }^{4}$ ${ }^{1}$ LCM - Laboratory of Catalysis and Materials - Associate Laboratory LSRE-LCM, Faculdade de Engenharia da Universidade do Porto, Porto, Portugal / Polytechnic Institute of Bragança, Bragança, Portugal (raquel.rodrigues@ipb.pt); ${ }^{2}$ University of Minho, Mechanical Engineering Department, Guimarães, Portugal / CEFT, Faculdade de Engenharia da Universidade do Porto, Porto, Portugal / Polytechnic Institute of Bragança, Bragança, Portugal (ruimec@ipb.pt); ${ }^{3}$ LCM - Laboratory of Catalysis and Materials - Associate Laboratory LSRE-LCM, Faculdade de Engenharia da Universidade do Porto, Porto, Portugal / Polytechnic Institute of Bragança, Bragança, Portugal (htgomes@ipb.pt); ${ }^{4} \mathrm{LCM}$ - Laboratory of Catalysis and Materials - Associate Laboratory LSRE-LCM, Faculdade de Engenharia da Universidade do Porto, Porto, Portugal (adrian@fe.up.pt)
\end{abstract}

\begin{abstract}
The amount of applications associated with microfluidic devices is increasing since the introduction of Lab-on-a-chip devices in the 1990s, especially regarding biomedical and clinical fields. However, in order for this technology to leave the fundamental research and become a day-life technology (e.g., as point-of-care testing), it needs to be disposable and reasonably less expensive. Polymers, due to their several advantages, such as easier microfabrication and low-cost, fill these needs. Several methods are reported regarding microfabrication and, thus, the main aim of the present work is to provide an overview of the most relevant microfabrication techniques found in literature employing polymers, clarifying also the main advantages and disadvantages of each technique and especially considering their cost and time-consumption. Moreover, a future outlook of lowcost microfabrication techniques and standard methods is provided.
\end{abstract}

Subject Headings. Nanotechnology, Medical diagnosis, Hand tool.

Author Keywords. Microfabrication, PDMS, Lab-on-a-chip, Low-cost techniques.

\section{Introduction}

Microfluidic devices (Figure 1) can be defined as the set of technologies which handles and processes small fluid volumes (e.g., $\mu \mathrm{L}, \mathrm{nL}$, and $\mathrm{pL}$ ) through microchannels geometries, with dimension of tens to hundreds of micrometers, embedded in a chip (Halldorsson et al. 2015, Monošík and Angnes 2015, Sia and Whitesides 2003, Whitesides 2006).

Related with these characteristics, since small amount of reagents and samples are used, microfluidic devices are suitable for analytical purposes with other several advantages, such as short time for analysis, reduction of reagent costs, low fabrication cost, miniaturization, sensitivity, selectivity, repeatability, portability and biocompatibility (Monošík and Angnes 2015, Whitesides 2006). Furthermore, microfluidic devices can be used as an integrative multiple processes device, called as Lab-on-a-chip (LOC) or micro total analysis system ( $\mu$ TAS). This concept of "miniaturized total chemical analysis system", known in our days as $\mu$ TAS or LOC, was introduced by Andreas Manz and co-workers in 1990 (Manz, Graber and Widmer 1990), and since then, the scientific expectations on this technology and analytical possibilities have increased as analytical tool (Whitesides 2006) and also a tool capable to improve the global health (van Reenen et al. 2014, Yager et al. 2006). Some application examples at the 
laboratory scale are found in clinical diagnostics, as point-of-care testing (Do et al. 2008, Liu et al. 2014, Novo, Chu and Conde 2014), but also in environment monitoring (Jeong et al. 2014, Mehta et al. 2006, Sun et al. 2014), food industry (Fronczek, You and Yoon 2013, Zhang, Zuo and Ye), microelectronics (Catalano et al. 2014, Daikuzono et al. 2015) and in numerous biochemical and biological processes, such as analysis of blood samples (Lima et al. 2008), drug screening (Nason et al. 2011), cell counting and sorting (Pratt et al. 2011), cell culture studies (Shi, Liu and Chen 2011), polymerase chain reaction (PCR) (Pan et al. 2010), DNA sequencing (Paegel, Blazej and Mathies 2003), among many others.

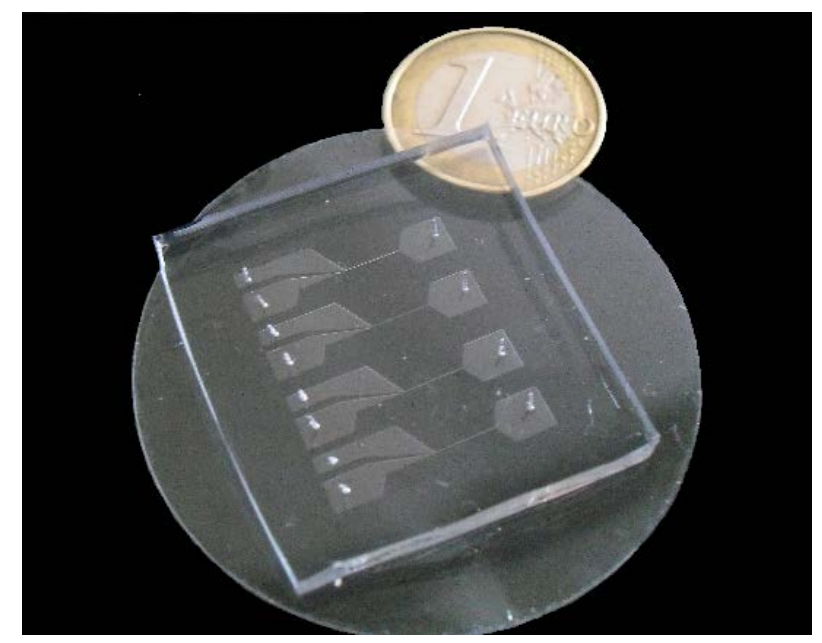

Figure 1. Microfluidic device designed for partial blood cells separation and deformability assessment.

However, to achieve future mass-market commercialization, it is crucial to find fabrication methods that allow their low cost production in large scale. This concern is especially important for medical applications, where the microdevices should be disposable, to avoid cross contamination or to be used in point-of-care testing (Attia, Marson and Alcock 2009).

Polymers, due to their several advantages (e.g., low-cost material, easier microfabrication over the other materials such as glass and silicon) are the most promising materials to fill the needs of mass-market utilization.

Due to the importance of the microfluidic field and the variety of microfabrication methods reported in literature, the present work intends to provide an overview of the most relevant techniques that use polymers, and to present recent low-cost techniques, that are of particular interest in research.

\section{Microfabrication techniques}

The first microfluidic device dates from 1979, when miniaturization of a gas chromatograph was developed at the Stanford University (Terry, Jerman and Angell 1979).

Similar to this first microfluidic device, most of the early systems were fabricated by microelectromechanical systems (MEMS) technology, due to the available semiconductor industry, highly developed in that time. Thus, techniques such as photolithography, thin film metallization and chemical etching on silicon and glass, were applied for the fabrication of these new type of devices (McDonald et al. 2000, Wu and Gu 2011).

Later, glass materials started to gain more interest, mainly due to the biocompatibility for applications in the biomedical field. However, the microfabrication difficulties in these glass materials, allied to the need of a cleanroom environment, high temperature requirement for sealing and expensive cost of glass materials, have hampered their wider application in microfluidics (Wu and Gu 2011). Due to these issues, a tremendous effort has been made since 
then, in order to find alternative materials that allow a more cost-effective production and easier microfabrication.

Polymers, due to its characteristics, fill the above needs and bring other advantages, such as good optical transparency, biocompatibility, chemical and mechanical properties as well as friendly system integration (e.g., interconnection with inlets/outlets) (Wu and Gu 2011). Therefore, these materials enable a high volume of production with good reproduction, lower cost of fabrication and also versatility in the design. These properties are of utmost importance to the creation of disposable microfluidic chips for biomedical and clinical applications. Nevertheless, polymers have some limitations regarding their properties or processing techniques in comparison to glass, such as limited operation-temperature range, higher autofluorescence and limited surface modification techniques (Attia, Marson and Alcock 2009).

For a better perception of the main characteristics of polymers and glass, Table 1 shows a comparison of properties that are important for the fabrication of microfluidic devices and to the selection of the microdevice substrate.

\begin{tabular}{|c|c|c|}
\hline Properties & Polymers & Glass \\
\hline Manufacturing cost & $\begin{array}{l}\text { Low manufacturing cost, especially for } \\
\text { mass-production when compared to } \\
\text { glass. }\end{array}$ & $\begin{array}{l}\text { Higher manufacturing cost related to } \\
\text { substrates and cleanroom facilities. }\end{array}$ \\
\hline $\begin{array}{l}\text { Complexity of } \\
\text { Fabrication }\end{array}$ & $\begin{array}{l}\text { Simpler than glass. Do not need wet } \\
\text { chemistry. }\end{array}$ & $\begin{array}{l}\text { Fabrication steps are more time } \\
\text { consuming and expensive. Wet } \\
\text { chemistry is used. }\end{array}$ \\
\hline $\begin{array}{l}\text { Operation } \\
\text { temperature }\end{array}$ & $\begin{array}{l}\text { Limited temperatures range due to } \\
\text { relatively low } \mathrm{T}_{\mathrm{g}} \text { (glass transition } \\
\text { temperature) compared to glass. }\end{array}$ & $\begin{array}{l}\text { Wide range of working temperature } \\
\text { compared to polymers. }\end{array}$ \\
\hline $\begin{array}{l}\text { Optical properties } \\
\text { and detection of } \\
\text { fluorescence }\end{array}$ & $\begin{array}{c}\text { In general, lower optical transparency } \\
\text { and higher autofluorescence compared to } \\
\text { glass. }\end{array}$ & Excellent optical properties. \\
\hline Bonding & $\begin{array}{c}\text { Many bonding options are available, } \\
\text { such as adhesives, thermal fusion, } \\
\text { ultrasonic welding and mechanical } \\
\text { clamping. }\end{array}$ & $\begin{array}{l}\text { Bonding options are more time } \\
\text { consuming than polymers and include } \\
\text { thermal, adhesive and anodic bonding. }\end{array}$ \\
\hline $\begin{array}{l}\text { Compatibility with } \\
\text { organic solvents, } \\
\text { strong acids and } \\
\text { bases }\end{array}$ & $\begin{array}{l}\text { In general polymers are not resistant to } \\
\text { most organic solvents or strong acids or } \\
\text { bases. }\end{array}$ & $\begin{array}{l}\text { Good resistance to organic solvents and } \\
\text { acids. }\end{array}$ \\
\hline Gas permeability & High gas permeability. & $\begin{array}{l}\text { Absence of gas permeability which is } \\
\text { required for some biological and cell } \\
\text { culture applications. }\end{array}$ \\
\hline Design & $\begin{array}{l}\text { Polymer fabrication techniques are more } \\
\text { flexible to complex geometries, such as } \\
\text { different cross-sections, heights and } \\
\text { higher aspect ratio square channels. }\end{array}$ & $\begin{array}{l}\text { Limited to simple designs (2D) due to } \\
\text { the nature of the etching process. }\end{array}$ \\
\hline
\end{tabular}

Table 1. Comparison of properties between polymers and glass for the fabrication of microfluidic devices. Adapted from (Attia, Marson and Alcock 2009)

The most popular polymers used to fabricate microfluidic devices are poly(methyl methacrylate) (PMMA), cyclic olefin copolymer (COC), poly(styrene) (PS), poly(carbonate) (PC), poly(ethyleneterephthalate glycol) (PETG) and poly(dimethylsiloxane) (PDMS) (Becker and Locascio 2002, Fiorini and Chiu 2005, Li et al. 2008, Sollier et al. 2011, Wu and Gu 2011).

Table 2 shows a comparison of the main properties between these polymers and the typical fabrication techniques that are applied. 


\begin{tabular}{|c|c|c|c|}
\hline Polymer & Main characteristics & Fabrication techniques & References \\
\hline PMMA & $\begin{array}{l}\text { Thermoplastic. Transparent. UV } \\
\text { resistance. Low water absorption. } \\
\text { Good abrasion resistance. }\end{array}$ & $\begin{array}{l}\text { Injection moulding. Hot } \\
\text { embossing. Laser } \\
\text { photoablation. X-ray } \\
\text { lithography. }\end{array}$ & $\begin{array}{l}\text { (Attia, Marson and Alcock } \\
\text { 2009, Becker and Locascio } \\
\text { 2002, Fiorini and Chiu } \\
\text { 2005, Li et al. 2008) }\end{array}$ \\
\hline $\mathrm{COC}$ & $\begin{array}{l}\text { Thermoplastic with high } \\
\text { transparency. High heat } \\
\text { resistance. Low water absorption. } \\
\text { High stiffness and strength. }\end{array}$ & $\begin{array}{l}\text { Injection moulding. } \\
\text { Hot embossing. }\end{array}$ & $\begin{array}{c}\text { (Attia, Marson and Alcock } \\
\text { 2009, Fiorini and Chiu } \\
\text { 2005, Khanarian and } \\
\text { Celanese 2001, Li et al. } \\
\text { 2008) }\end{array}$ \\
\hline PS & $\begin{array}{l}\text { Thermoplastic. Excellent } \\
\text { electrical properties. Resistant to } \\
\text { a wide variety of chemicals. }\end{array}$ & $\begin{array}{l}\text { Injection moulding. } \\
\text { Hot embossing. Laser } \\
\text { photoablation. }\end{array}$ & $\begin{array}{l}\text { (Becker and Locascio } \\
\text { 2002, Fiorini and Chiu } \\
\text { 2005, Li et al. 2008) }\end{array}$ \\
\hline PC & $\begin{array}{l}\text { Transparent thermoplastic. High } \\
\text { heat resistance. High stiffness } \\
\text { and strength. }\end{array}$ & $\begin{array}{l}\text { Injection moulding. } \\
\text { Hot embossing. Laser } \\
\text { photoablation. }\end{array}$ & $\begin{array}{c}\text { (Attia, Marson and Alcock } \\
\text { 2009, Becker and Locascio } \\
\text { 2002, Fiorini and Chiu } \\
\text { 2005, Li et al. 2008) }\end{array}$ \\
\hline PTEG & $\begin{array}{l}\text { Transparent thermoplastic. Good } \\
\text { impact and chemical resistance. }\end{array}$ & $\begin{array}{l}\text { Hot embossing. Laser } \\
\text { photoablation. }\end{array}$ & $\begin{array}{l}\text { (Becker and Locascio } \\
\text { 2002, Fiorini and Chiu } \\
\text { 2005) }\end{array}$ \\
\hline PDMS & $\begin{array}{l}\text { Transparent elastomeric polymer. } \\
\text { Biocompatibility. High flexibility. } \\
\text { High gas permeability. UV } \\
\text { resistance. Chemically inert. } \\
\text { Thermally stable. }\end{array}$ & $\begin{array}{l}\text { Soft-lithography. Direct } \\
\text { laser plotting. }\end{array}$ & $\begin{array}{l}\text { (Becker and Locascio } \\
\text { 2002, Fiorini and Chiu } \\
\text { 2005, Mata, Fleischman } \\
\text { and Roy 2005, McDonald } \\
\text { et al. 2000) }\end{array}$ \\
\hline
\end{tabular}

Table 2. Comparison of the properties and fabrication techniques between the most popular polymers reported in literature for the fabrication of microfluidic devices

The selection of the polymer to be applied as a matrix in a microfluidic device has to be highly related with the required properties of the fabrication method (e.g., PMMA is an option for injection moulding, which requires a thermoplastic polymer), and also with properties for which it was designed (e.g., PDMS is a good choice if it requires a biocompatible polymer for culture cells applications).

Currently, there are a variety of methods regarding the fabrication of microfluidic devices, including injection moulding (Attia, Marson and Alcock 2009), hot embossing (Becker and Heim 2000), soft-lithography (Lima et al. 2008), direct laser plotting (Wang et al. 2012), laser photoablation or laser micromachining (Rossier, Reymond and Michel 2002), photolithography (Marchesan et al. 2013), X-ray lithography (Mappes, Achenbach and Mohr 2007), among other more recently and low-costly microfabrication techniques, such as the print-and-peel techniques, e.g., xurography (Pinto et al. 2014).

The following sub-chapters will focus on the most used techniques described in literature to fabricate microfluidic devices from polymer substrates, and also the recent low-cost techniques that are gaining interest, especially at research level.

\subsection{Hot embossing}

Hot embossing was first described in the late 1990s and some of the imprinting plastic subtracts methods are still used today (Becker and Locascio 2002). The embossing is a technique involving thermoplastic materials, such as PMMA, PC COC, PS or PETG, which are patterned against a master (stamp), which is normally silicon or metal sheets, using pressure and heat (Fiorini and Chiu 2005, Sollier et al. 2011, Becker and Locascio 2002). Although embossing is a fast and inexpensive technique, it requires dedicated press equipment and a 
robust mould, which can be time-consuming, and thus not ideal for routine microfluidic designs, such as for laboratory testing (Fiorini and Chiu 2005).

\subsection{Injection moulding}

Injection moulding is a process commonly used in the plastics industry to produce a large variety of everyday objects.

This technique was first described in 1997 by researchers at Soane Bioscience for the fabrication of microchannel devices regarding the electrophoretic separation of DNA (McCormick et al. 1997).

The injection moulding process starts with thermoplastic pellets, such as PMMA or PC, being melted and injected under high pressure into the heated mould cavity. The injected pieces are then cooled and released from the mould (Fiorini and Chiu 2005, Sollier et al. 2011). Similar to hot embossing, injection moulding is mostly applied in industry, due to the complexity of moulding equipment and fabrication of the masters, as well as initial cost of the moulding equipment and masters. However, when compared with embossing, this process offers a highthroughput fabrication option, with large-volume production (Fiorini and Chiu 2005).

\subsection{Laser photoablation}

Photoablation was first reported in the literature as a method to fabricate microfluidic channels, by Roberts and co-workers in 1997 (Roberts et al. 1997). This technique involves the use of a high-powered pulsed laser to remove material from a sheet of thermoplastic material (Fiorini and Chiu 2005). In this process, a shock wave is produced while particles are injected from the substrate, creating the microchannels geometries (Becker and Locascio 2002).

Micromachining, using a laser ablation, can be achieved either by exposing the polymer substrate with a mask that defines the area to be ablated, or using a direct-write by a maskless process (Becker and Locascio 2002). The depth of the ablated channels is dependent on the pulse rate, as well as on the substrate characteristics. ArF excimer lasers (193 $\mathrm{nm}$ ) have been used to ablate PS, PC and polyethyleneterephthalate (PET), while KrF excimer lasers (248 nm) have been used to PMMA, PETG, PS, PC, among others (Becker and Locascio 2002, Fiorini and Chiu 2005). $\mathrm{CO}_{2}$ lasers with wavelengths in the infrared region $(10.6 \mu \mathrm{m})$ have also been applied to PMMA or PET (Fiorini and Chiu 2005).

The main advantage of this technique, for the fabrication of microfluidic devices, is that new microfluidic designs are easily programmed into the system using a direct-write process. However, this direct-write laser ablation approach has several limitations concerning massproduction (Fiorini and Chiu 2005).

\subsection{Soft-lithography}

The term "soft-lithography" was given by Xia and Whitesides in 1998 (Xia and Whitesides 1998), as a set of techniques that includes replica moulding using elastomeric materials for the fabrication of microfluidic devices, specially PDMS, as well as for the patterning of surfaces using PDMS stamps (Fiorini and Chiu 2005).

Similar to all the other methods described so far, a master template/mask is necessary to replicate the moulds. To date, the most current mask fabrication technique used for softlithography is photolithography, due to the high resolution of the photolithographic masks, enabling the fabrication of microchannels with a few nanometers, as well as complex geometries. Thereby, after the silicon master has been made, an elastomeric polymer is casted onto silicon stamp and cured. The curing process may be performed at room temperature or at slightly elevated temperature to speed the curing process (PDMS is normally cured in the 
range of temperature between 40 and $80 \stackrel{\circ}{\circ}$, during 20 min to $2 \mathrm{~h}$ ). Finally, after the PDMS is cured the moulds are peeled off from the mask. The same process may be repeated to obtain hundreds of replicas.

The main advantage of this technique is the ease bonding of the PDMS moulds to plastic, elastomeric polymers or glass substrates regarding the sealing process, which can be reversible or irreversible depending on the applied sealing process. Moreover, the simplicity associated with this easy sealing procedure, has made this fabrication technology one of the most widely used in the prototyping of microfluidic systems (Becker and Locascio 2002). Indeed, soft-lithography technique using PDMS, brings several advantages including low cost, fast processing, reusability of the masters, design of complex 3-dimensional systems by multilayer fabrication, excellent optical transparency, easy installation of fluidic interconnects, as well as the applicability to a variety of biological and cellular processes due to the biocompatibility property of the polymer (Fiorini and Chiu 2005).

Regarding the elastomeric polymers used in this technique for fabrication of microfluidic devices, the majority of reports in the literature used PDMS (Becker and Locascio 2002). Nevertheless, other elastomeric polymers can also be suitable for moulding by the softlithography technique.

\subsection{X-ray lithography}

More recently, X-ray lithography is being applied for the fabrication of microfluidic channels with complex 3D structures (Romanato et al. 2004), normally obtained by layer-to-layer with lithography techniques, as well as for submicron feature size and high aspect ratios (thickness/minimum feature size, AR) (Mappes, Achenbach and Mohr 2007).

The fabrication process starts with a quartz-chrome mask generated to define the pattern. Then, a reusable gold/Kapton ${ }^{\mathrm{TM}}$ mask for the LIGA process (German acronym for "LIthographie Galvanoformung Adformung", which means, lithography, electroplating and moulding) is also generated by coating a Kapton film with a very thin film of gold placed in contact with PMMA substrate. The Kapton layer (transparent to X-rays) is then coated with photoresist and the image from the quartz-chrome mask transferred photolithographically to the photoresist over the Kapton layer. After this process, a thick layer of gold is deposited onto the Kapton surface in the open areas of the photoresist. The X-rays are absorbed by the gold layer while the section of the Kapton without the thick gold layer is transparent to the X-rays. After that, the photoresist is removed and the polymer substrate irradiated through the gold/Kapton mask in order to degrade the exposed polymer. Finally, the degraded polymer is dissolved in a solvent that solubilizes the reaction products forming the microstructures to yield high aspect ratio structures with straight and smooth walls (Becker and Locascio 2002, Mappes, Achenbach and Mohr 2007, Romanato et al. 2004).

\subsection{Xurography}

The fabrication of microfluidic devices generally requires a mask, which will serve as mould for manufacturing the microchips.

The most popular and traditional technique for the development of this mask, is photolithography due to its main advantages, such as high-resolution capabilities, low material costs, gas permeability and optical transparency (Pinto et al. 2014, Duffy et al. 1998). However, this technique requires cleanroom environment and specialized equipment and operators, making the process expensive and also time-consuming. These drawbacks, are creating a need for the alternative low-cost techniques, especially to be applied in research institutions without dedicated facilities (Pinto et al. 2014). Furthermore, these low-cost 
techniques can reduce the time required for the design and testing of new microchip designs brought from research ideas.

Concerning this need, new nonlithographic techniques, such as xurography are gaining interest in the scientific field.

The first report using a xerographic process to produce microfluidic devices was published in 2001, by Tan and co-workers (Tan et al. 2001). This novel print-and-peel (PAP) technique, has shown to be effective and most important of all, a rapid and low-cost technique to fabricate microfluidic channels.

Xurography, uses a cutting plotter machine and adhesive foils (normally, vinyl films, but can be also performed with PET, nitrocellulose and aluminium) to generate the master moulds or mask (Focke et al. 2010, Pinto et al. 2014). The mask is moulded with elastomeric polymers, such as PDMS used in the soft-lithography technique. Moreover, this technique can be directly applied to fabricate microchannels. In both ways, this technique does not involve photolithography or cleanroom facilities, which is a great advantage.

The main disadvantage of this technique is the relatively poor resolution capabilities and micron-sizes precision. The thickness of the microchannel is also dependent on the type of foil material that is used.

\subsection{Other low-cost fabrication techniques}

Other low-cost technologies, including laser direct machining (Wang et al. 2012) or 3D printing (Erkal et al. 2014, McDonald et al. 2002), are also being used to the fabrication of microfluidic devices.

Laser direct machining, also known as direct laser plotting consists in a laser technique that was adapted to generate microchannels directly into the substrate of the microfluidic devices, normally cured PDMS or PMMA, without the need of a mask.

To create the patterns, four parameters can be adjusted, namely, laser power, pulse density (pulses per inch-PPI), focus and laser moving speed (Wang et al. 2012). This fabrication technique offers advantages such as time and cost saving over the conventional softlithography technique, eliminating the need of a cleanroom facility, as well as complex fabrication steps. Due to these advantages, laser direct machining appears to be suitable to be applied in research laboratories. However, it needs improvement in order to be useful in mass-production.

$3 D$ printing, is an adapted technique that has been recently applied to produce microfluidic devices. This techniques operates printers either by printing a thermoplastic polymer that solidifies after extrusion or by printing a binding material that joins regions of a predeposited layer (McDonald et al. 2002). Generally, the extruded thermoplastic material serves as the 3Dmask, which is further used to prototype the microfluidic devices.

Although this fabrication technique has some limitations concerning the size of the microchannels and some laborious fabrication steps for the final microfluidic devices, the further development of this 3D-printing technique will certainly become a major theme in the fabrication of microfluidic devices, due to their several advantages, such the design of complex 3D structures and rapid prototyping.

\section{Discussion and future outlook}

In the 1980s, microfluidic devices were first developed using the available and highly established fabrication techniques brought from the MEMS technology that used glass and silicon as substracts (Terry, Jerman and Angell 1979). Since then, microfabrication techniques 
have greatly evolved alongside with science materials, especially with the development of polymers. Actually, due to their many advantages, such as being a low-cost material, easier microfabrication and having a wide range of mechanical and chemical properties, polymers are perfect materials to be used in the fabrication of microfluidic devices and became, as envisioned by Whitesides (Whitesides 2006), the major theme in analysis.

Furthermore, the characteristics of polymers overcome the major issue of biomedical and clinical applications, which is the creation of a low-cost and disposable microfluidic chip, to be used as point-of-care testing.

Indeed, there are several methods reported in the literature for the fabrication of microfluidic devices using polymers as a substrate. Usually, the selection of the fabrication method will highly depend on the final goal, which for the industrial perspective, is the mass production while for a research laboratory level, is the rapid prototyping, which includes short fabrication time and low-cost for a complete cycle from design to testing (Sollier et al. 2011).

Due to these two different perspectives, industrial and laboratory level, the main advantages and disadvantages of each fabrication technique described are compiled in Table 3 for summary purposes.

\begin{tabular}{|c|c|c|}
\hline Methods & Advantages & Disadvantages \\
\hline Hot embossing & $\begin{array}{l}\text { Precise and rapid in the replication of } \\
\text { microstructures. Mass production. }\end{array}$ & $\begin{array}{l}\text { Restricted to thermoplastics. Time- } \\
\text { consuming. Complex 3D structures are } \\
\text { difficult to be fabricated. }\end{array}$ \\
\hline Injection moulding & $\begin{array}{l}\text { Mass production. Fine features. Low } \\
\text { cycle time. Highly automated. }\end{array}$ & $\begin{array}{l}\text { Restricted to thermoplastics. High cost } \\
\text { mould. Micro size precision is limited. }\end{array}$ \\
\hline Laser photoablation & Rapid. Large format production. & $\begin{array}{l}\text { Limited materials. Multiple treatment } \\
\text { session. Difficulties for mass production. } \\
\text { Micro size precision is limited. }\end{array}$ \\
\hline Soft-lithography & $\begin{array}{l}\text { High-resolution and 3D geometries. } \\
\text { Cost-effective. Excellent micro size } \\
\text { precision. }\end{array}$ & $\begin{array}{l}\text { Pattern deformation and vulnerability to } \\
\text { defects. Difficult to fabricate circular } \\
\text { geometries. }\end{array}$ \\
\hline X-ray lithography & $\begin{array}{l}\text { High-resolution. Straight and smooth } \\
\text { walls. }\end{array}$ & $\begin{array}{l}\text { Complex and difficult master fabrication. } \\
\text { Time consuming and high cost process. }\end{array}$ \\
\hline Xurography & Low-cost and rapid technique. & $\begin{array}{l}\text { Complex 3D structures are difficult to be } \\
\text { fabricated. Micro size precision is limited. }\end{array}$ \\
\hline Direct laser plotting & $\begin{array}{l}\text { Low-cost and rapid technique. Free- } \\
\text { mask technique. }\end{array}$ & $\begin{array}{l}\text { Complex 3D structures are difficult to be } \\
\text { fabricated. Micro size precision is limited. } \\
\text { Reproducibility of the microdevices. }\end{array}$ \\
\hline 3D-printing & Low-cost and rapid technique. & $\begin{array}{l}\text { Multiple treatment session. Difficulties for } \\
\text { mass production. Micro size precision is } \\
\text { limited. }\end{array}$ \\
\hline
\end{tabular}

Table 3. Main advantages and disadvantages for the fabrication techniques of the microfluidic devices based in polymer substrates. Adapted from (Wu and Gu 2011)

In a mass-production industrial perspective, hot embossing and injection moulding are in generally the most used microdevice fabrication methods. Beyond the initial cost of equipments and mask moulds, the mass-production with high precision, short replication time and the very long lifetime of the stamps, makes these techniques very attractive for industrial purposes. Therefore, these techniques are more commonly found in industrial applications than in research laboratories. 
On the other hand, research laboratories, focused their needs in rapid prototyping of microfluidic devices (as new ideas have to be quickly tested and improved), as well as in a low cost fabrication perspective.

Figure 2 shows an overview of the fabrication methods previously presented in Table 3, in a time and cost perspective.

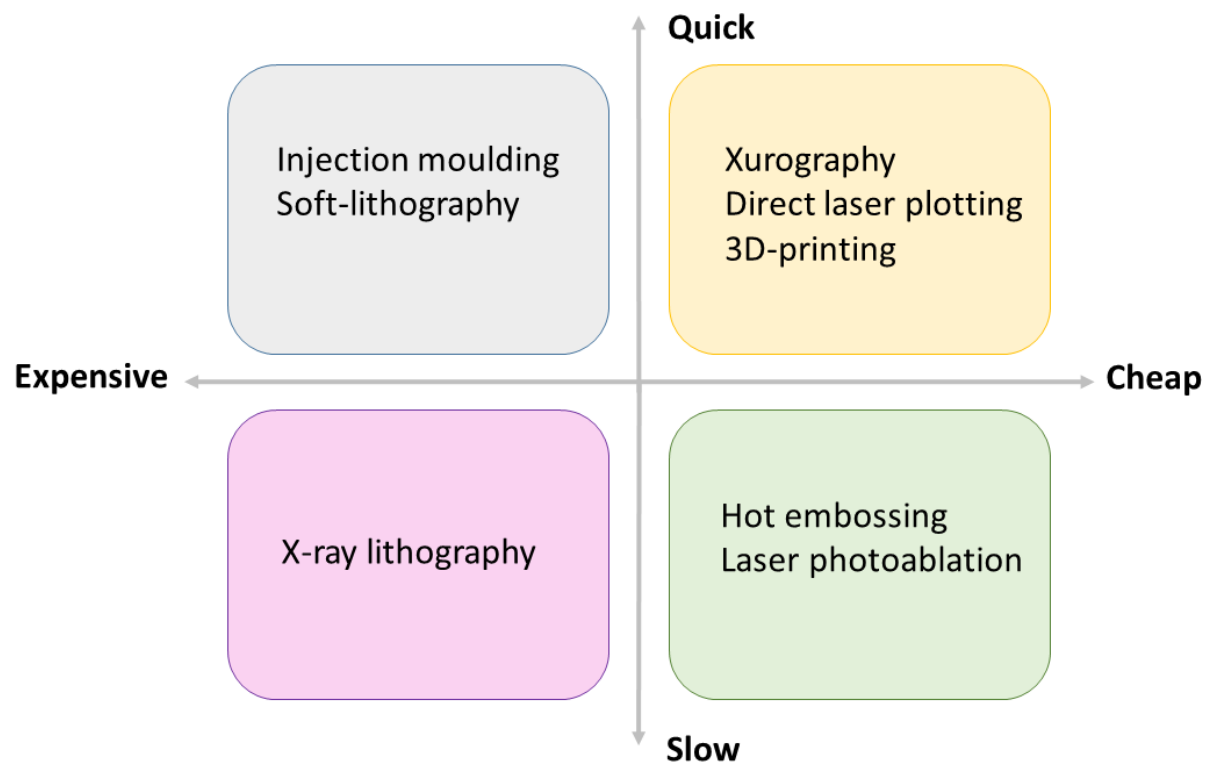

Figure 2. Overview of microfluidic fabrication techniques regarding the costs and time-consumption point-of-view

Under a research laboratory point-of-view, where the production cost and time-consumption are of utmost importance, Figure 2 clearly reveals that the recent low-cost techniques are filling a gap in the microdevice fabrication methods, concerning the rapid and inexpensive microchips for testing new ideas.

Nevertheless, it is important to bear in mind that for the research laboratory the available technologies, equipments and preferred material substrates, are also crucial issues for the selection of the microfabrication method.

Indeed, these last concerns are the main cause for the recent development of low-cost microfabrication techniques, such as xurography, direct laser plotting or even 3D-printing (there are many others).

Actually, low-cost techniques generally avoid the use of cleanroom facilities or lithographic techniques to produce a mask, or even the complete microdevice. Due to these specifications, low-cost fabrication techniques are becoming an important theme in the field, allowing a quick rise of studies and achievements in many scientific domains by spreading the fabrication of microfluidic devices to almost any research laboratory, as it can be observed in Figure 3, where it is shown a metadata analysis made in Scopus database with the search sentence, "low-cost fabrication for microfluidic devices", between 1996 (year of the first work reported in literature) and 2014. 


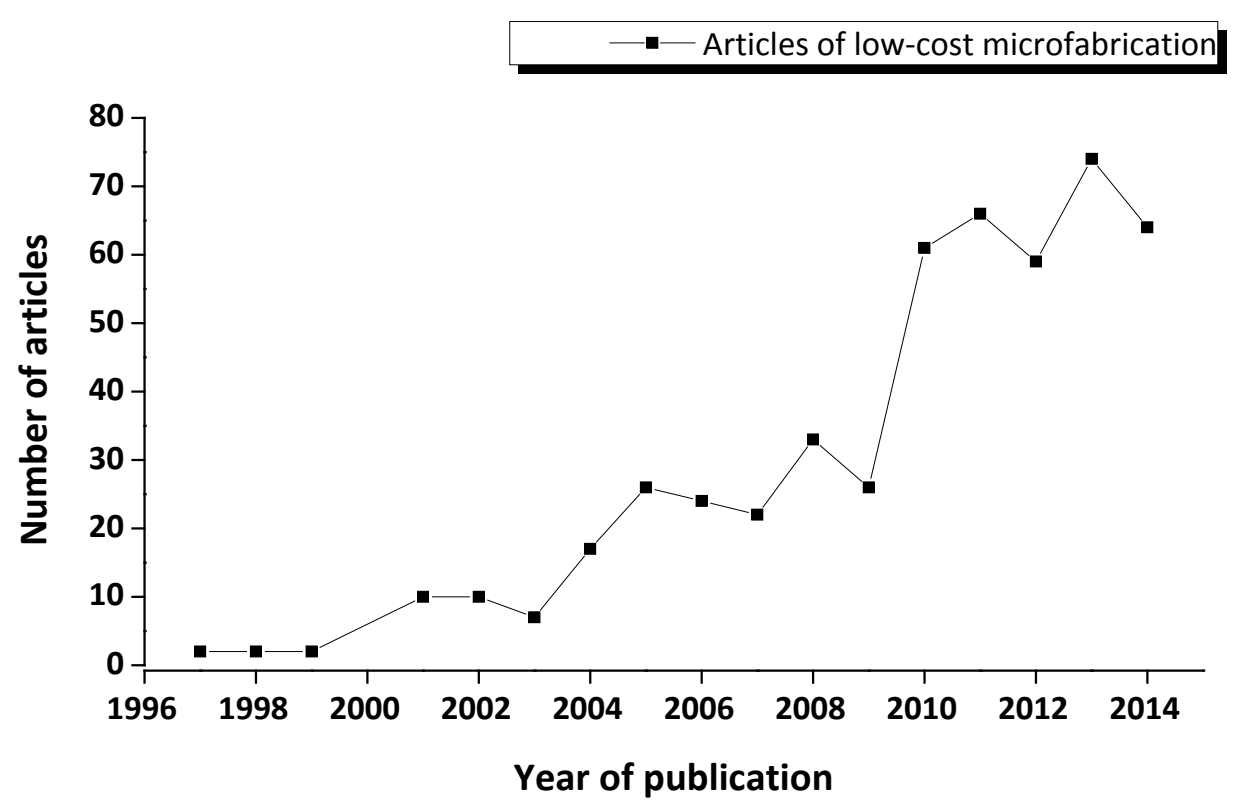

Figure 3. Number of publications concerning the low-cost microfabrication techniques since 1996 to 2014

The exponential growth of published documents concerning low-cost fabrication techniques, mainly articles, conference papers and reviews, is shown in Figure 3. Mainly after 2009 where the number of published documents have triplicated, proving the growing interest from the scientific community for these alternative and low-cost microfabrication techniques.

However, these recent low-cost techniques still need improvements, especially concerning the micro size precision, complex 3-D microchannels designs and reproducibility of the microdevices. Nevertheless, and despite these limitations, low-cost techniques are still representing for research laboratories an important tool to be used as screening experiments, in order to test, discard and improve microchannels designs that can be further developed with other more traditional and improved microfabrication techniques, such as photolithography or even X-ray lithography.

Considering the three past decades, it seems to be obvious that with the growing interest for low-cost microfabrication techniques by the scientific community, the limitations will be overcome in a near future. Therefore, these techniques will probably became a major theme concerning the fabrication techniques for microfluidic devices having a massive impact in the worldwide utilization of microdevices in many scientific fields.

\section{References}

Attia, Usama M, Silvia Marson and Jeffrey R Alcock. 2009. "Micro-injection moulding of polymer microfluidic devices." Microfluidics and Nanofluidics no. 7 (1):1-28. DOI: 10.1007/s10404-009-0421-x.

Becker, Holger and Ulf Heim. 2000. "Hot embossing as a method for the fabrication of polymer high aspect ratio structures." Sensors and Actuators A: Physical no. 83 (1-3):130-135. DOI: 10.1016/S0924-4247(00)00296-X.

Becker, Holger and Laurie E. Locascio. 2002. "Polymer microfluidic devices." Talanta no. 56 (2):267287. DOI: S0039-9140(01)00594-X.

Catalano, Rossella, Gerardo Perozziello, Giuseppina Simone, Patrizio Candeloro, Francesco Gentile, Maria Laura Coluccio, Francesca Pardeo, Manfred Burghammer, Giovanni Cuda, Christian Riekel and Enzo Di Fabrizio. 2014. "Optimized fabrication protocols of microfluidic devices for X-ray analysis." Microelectronic Engineering no. 124:13-16. DOI: 10.1016/j.mee.2014.04.016. 
Daikuzono, Cristiane M., Cleber A. R. Dantas, Diogo Volpati, Carlos J. L. Constantino, Maria H. O. Piazzetta, Angelo L. Gobbi, David M. Taylor, Osvaldo N. Oliveira Jr and Antonio Riul Jr. 2015. "Microfluidic electronic tongue." Sensors and Actuators B: Chemical no. 207, Part B:1129-1135. DOI: 10.1016/j.snb.2014.09.112.

Do, Jaephil, Sehwan Lee, Jungyup Han, Junhai Kai, Chien-Chong Hong, Chuan Gao, Joseph H. Nevin, Gregory Beaucage and Chong H. Ahn. 2008. "Development of functional lab-on-a-chip on polymer for point-of-care testing of metabolic parameters." Lab on a Chip no. 8 (12):2113-2120. DOI: 10.1039/B811169C.

Duffy, David C., J. Cooper McDonald, Olivier J. A. Schueller and George M. Whitesides. 1998. "Rapid Prototyping of Microfluidic Systems in Poly(dimethylsiloxane)." Analytical Chemistry no. 70 (23):4974-4984. DOI: 10.1021/ac980656z.

Erkal, Jayda L., Asmira Selimovic, Bethany C. Gross, Sarah Y. Lockwood, Eric L. Walton, Stephen McNamara, R. Scott Martin and Dana M. Spence. 2014. "3D printed microfluidic devices with integrated versatile and reusable electrodes." Lab on a Chip no. 14 (12):2023-2032. DOI: 10.1039/C4LC00171K.

Fiorini, G. S. and D. T. Chiu. 2005. "Disposable microfluidic devices: fabrication, function, and application." Biotechniques no. 38 (3):429-46. http://www.ncbi.nlm.nih.gov/pubmed/15786809.

Focke, Maximilian, Dominique Kosse, Claas Muller, Holger Reinecke, Roland Zengerle and Felix von Stetten. 2010. "Lab-on-a-Foil: microfluidics on thin and flexible films." Lab on a Chip no. 10 (11):1365-1386. DOI: 10.1039/C001195A.

Fronczek, Christopher F., David J. You and Jeong-Yeol Yoon. 2013. "Single-pipetting microfluidic assay device for rapid detection of Salmonella from poultry package." Biosensors and Bioelectronics no. 40 (1):342-349. DOI: 10.1016/j.bios.2012.07.076.

Halldorsson, Skarphedinn, Edinson Lucumi, Rafael Gómez-Sjöberg and Ronan M. T. Fleming. 2015. "Advantages and challenges of microfluidic cell culture in polydimethylsiloxane devices." Biosensors and Bioelectronics no. 63:218-231. DOI: 10.1016/j.bios.2014.07.029.

Jeong, Heon-Ho, Seong-Geun Jeong, Aeri Park, Sung-Chan Jang, Soon Gyu Hong and Chang-Soo Lee. 2014. "Effect of temperature on biofilm formation by Antarctic marine bacteria in a microfluidic device." Analytical Biochemistry no. 446:90-95. DOI: 10.1016/j.ab.2013.10.027.

Khanarian, G. and Hoechst Celanese. 2001. "Optical properties of cyclic olefin copolymers." Optical Engineering no. 40 (6):1024-1029. DOI: 10.1117/1.1369411.

Li, Shiguang, Zhiguang Xu, Aaron Mazzeo, Daniel J. Burns, Gang Fu, Matthew Dirckx, Vijay Shilpiekandula, Xing Chen, Nimai C. Nayak, Eehern Wong, Soon Fatt Yoon, Zhong Ping Fang, Kamal Youcef-Toumi, David Hardt, Shu Beng Tor, Chee Yoon Yue and Jung-Hoon Chun. 2008. "Review of production of microfluidic devices: material, manufacturing and metrology." no. 6993:69930F69930F-12. DOI: 10.1117/12.781942.

Lima, R., S. Wada, S. Tanaka, M. Takeda, T. Ishikawa, K. Tsubota, Y. Imai and T. Yamaguchi. 2008. "In vitro blood flow in a rectangular PDMS microchannel: experimental observations using a confocal micro-PIV system." Biomed Microdevices no. 10 (2):153-67. DOI: 10.1007/s10544-007-9121-z.

Liu, Jikun, Bingchen Du, Panhe Zhang, Mohan Haleyurgirisetty, Jiangqin Zhao, Viswanath Ragupathy, Sherwin Lee, Don L. DeVoe and Indira K. Hewlett. 2014. "Development of a microchip Europium nanoparticle immunoassay for sensitive point-of-care HIV detection." Biosensors and Bioelectronics no. 61:177-183. DOI: 10.1016/j.bios.2014.04.057.

Manz, A., N. Graber and H. M. Widmer. 1990. "Miniaturized total chemical analysis systems: A novel concept for chemical sensing." Sensors and Actuators B: Chemical no. 1 (1-6):244-248. DOI: 10.1016/0925-4005(90)80209-I.

Mappes, Timo, Sven Achenbach and Juergen Mohr. 2007. "X-ray lithography for devices with high aspect ratio polymer submicron structures." Microelectronic Engineering no. 84 (5-8):1235-1239. DOI: 10.1016/j.mee.2007.01.154. 
Marchesan, Silvia, Christopher D. Easton, Katie E. Styan, Patrick Leech, Thomas R. Gengenbach, John S. Forsythe and Patrick G. Hartley. 2013. "SU-8 photolithography on reactive plasma thin-films: coated microwells for peptide display." Colloids and Surfaces B: Biointerfaces no. 108:313-321. DOI: 10.1016/j.colsurfb.2013.03.018.

Mata, Alvaro, AaronJ Fleischman and Shuvo Roy. 2005. "Characterization of Polydimethylsiloxane (PDMS) Properties for Biomedical Micro/Nanosystems." Biomedical Microdevices no. 7 (4):281-293. DOI: 10.1007/s10544-005-6070-2.

McCormick, R. M., R. J. Nelson, M. G. Alonso-Amigo, D. J. Benvegnu and H. H. Hooper. 1997. "Microchannel electrophoretic separations of DNA in injection-molded plastic substrates." Analytical Chemistry no. 69 (14):2626-30. DOI: 10.1021/ac9701997.

McDonald, J. C., D. C. Duffy, J. R. Anderson, D. T. Chiu, H. Wu, O. J. Schueller and G. M. Whitesides. 2000. "Fabrication of microfluidic systems in poly(dimethylsiloxane)." Electrophoresis no. 21 (1):2740. DOI: 10.1002/(sici)1522-2683(20000101)21:1<27::aid-elps27>3.0.co;2-c.

McDonald, J. Cooper, Michael L. Chabinyc, Steven J. Metallo, Janelle R. Anderson, Abraham D. Stroock and George M. Whitesides. 2002. "Prototyping of Microfluidic Devices in Poly(dimethylsiloxane) Using Solid-Object Printing." Analytical Chemistry no. 74 (7):1537-1545. DOI: 10.1021/ac010938q.

Mehta, A., H. Shekhar, S. H. Hyun, S. Hong and H. J. Cho. 2006. "A micromachined electrochemical sensor for free chlorine monitoring in drinking water." Water Sci Technol no. 53 (4-5):403-10. DOI: 10.2166/wst.2006.146.

Monošík, Rastislav and Lúcio Angnes. 2015. "Utilisation of micro- and nanoscaled materials in microfluidic analytical devices." Microchemical Journal no. 119:159-168. DOI: 10.1016/j.microc.2014.12.003.

Nason, F., E. Morganti, C. Collini, C. Ress, S. Bersini, G. Pennati, F. Boschetti, A. Colombini, G. Lombardi, G. Banfi, L. Lorenzelli and G. Dubini. 2011. "Design of microfluidic devices for drug screening on invitro cells for osteoporosis therapies." Microelectronic Engineering no. 88 (8):1801-1806. DOI: 10.1016/j.mee.2011.02.115.

Novo, P., V. Chu and J. P. Conde. 2014. "Integrated optical detection of autonomous capillary microfluidic immunoassays:a hand-held point-of-care prototype." Biosensors and Bioelectronics no. 57:284-291. DOI: 10.1016/j.bios.2014.02.009.

Paegel, Brian M., Robert G. Blazej and Richard A. Mathies. 2003. "Microfluidic devices for DNA sequencing: sample preparation and electrophoretic analysis." Current Opinion in Biotechnology no. 14 (1):42-50. DOI: 10.1016/S0958-1669(02)00004-6.

Pan, Xiaoyan, Lei Jiang, Kaiying Liu, Bingcheng Lin and Jianhua Qin. 2010. "A microfluidic device integrated with multichamber polymerase chain reaction and multichannel separation for genetic analysis." Analytica Chimica Acta no. 674 (1):110-115. DOI: 10.1016/j.aca.2010.06.005.

Pinto, Elmano, Vera Faustino, Raquel Rodrigues, Diana Pinho, Valdemar Garcia, João Miranda and Rui Lima. 2014. "A Rapid and Low-Cost Nonlithographic Method to Fabricate Biomedical Microdevices for Blood Flow Analysis." Micromachines no. 6 (1):121-135. DOI: 10.3390/mi6010121.

Pratt, Erica D., Chao Huang, Benjamin G. Hawkins, Jason P. Gleghorn and Brian J. Kirby. 2011. "Rare cell capture in microfluidic devices." Chemical Engineering Science no. 66 (7):1508-1522. DOI: 10.1016/j.ces.2010.09.012.

Roberts, Matthew A., Joël S. Rossier, Paul Bercier and Hubert Girault. 1997. "UV Laser Machined Polymer Substrates for the Development of Microdiagnostic Systems." Analytical Chemistry no. 69 (11):2035-2042. DOI: 10.1021/ac961038q.

Romanato, F., M. Tormen, L. Businaro, L. Vaccari, T. Stomeo, A. Passaseo and E. Di Fabrizio. 2004. "Xray lithography for 3D microfluidic applications." Microelectronic Engineering no. 73-74:870-875. DOI: 10.1016/j.mee.2004.03.067. 
Rossier, J., F. Reymond and P. E. Michel. 2002. "Polymer microfluidic chips for electrochemical and biochemical analyses." Electrophoresis no. 23 (6):858-67. DOI: 10.1002/15222683(200203)23:6<858::aid-elps858>3.0.co;2-3.

Shi, Jian, Li Liu and Yong Chen. 2011. "Investigation of cell culture in microfluidic devices with different bi-layer substrates." Microelectronic Engineering no. 88 (8):1693-1697. DOI: 10.1016/j.mee.2011.01.047.

Sia, S. K. and G. M. Whitesides. 2003. "Microfluidic devices fabricated in poly(dimethylsiloxane) for biological studies." Electrophoresis no. 24 (21):3563-76. DOI: 10.1002/elps.200305584.

Sollier, E., C. Murray, P. Maoddi and D. Di Carlo. 2011. "Rapid prototyping polymers for microfluidic devices and high pressure injections." Lab on a Chip no. 11 (22):3752-65. DOI: 10.1039/c1lc20514e.

Sun, Guoqiang, Panpan Wang, Shenguang Ge, Lei Ge, Jinghua Yu and Mei Yan. 2014. "Photoelectrochemical sensor for pentachlorophenol on microfluidic paper-based analytical device based on the molecular imprinting technique." Biosensors and Bioelectronics no. 56:97-103. DOI: 10.1016/j.bios.2014.01.001.

Tan, Aimin, Kenneth Rodgers, John P. Murrihy, Cian O'Mathuna and Jeremy D. Glennon. 2001. "Rapid fabrication of microfluidic devices in poly(dimethylsiloxane) by photocopying." Lab on a Chip no. 1 (1):7-9. DOI: 10.1039/B102905N.

Terry, S. C., J. H. Jerman and J. B. Angell. 1979. "A gas chromatographic air analyzer fabricated on a silicon wafer." Electron Devices, IEEE Transactions on no. 26 (12):1880-1886. DOI: 10.1109/TED.1979.19791.

van Reenen, Alexander, Arthur M. de Jong, Jaap M. J. den Toonder and Menno W. J. Prins. 2014. "Integrated lab-on-chip biosensing systems based on magnetic particle actuation - a comprehensive review." Lab on a Chip no. 14 (12):1966-1986. DOI: 10.1039/C3LC51454D.

Wang, Limu, Rimantas Kodzius, Xin Yi, Shunbo Li, Yu Sanna Hui and Weijia Wen. 2012. "Prototyping chips in minutes: Direct Laser Plotting (DLP) of functional microfluidic structures." Sensors and Actuators B: Chemical no. 168:214-222. DOI: 10.1016/j.snb.2012.04.011.

Whitesides, George M. 2006. "The origins and the future of microfluidics." Nature no. 442 (7101):368373. DOI: $10.1038 /$ nature05058.

Wu, Jing and Min Gu. 2011. "Microfluidic sensing: state of the art fabrication and detection techniques." Journal of Biomedical Optics no. 16 (8):080901-080901-12. DOI: 10.1117/1.3607430.

Xia, Younan and George M. Whitesides. 1998. "Soft Lithography." Angewandte Chemie International Edition no. 37 (5):550-575. DOI: 10.1002/(SICI)1521-3773(19980316)37:5<550::AID-ANIE550>3.0.CO;2-G.

Yager, Paul, Thayne Edwards, Elain Fu, Kristen Helton, Kjell Nelson, Milton R. Tam and Bernhard H. Weigl. 2006. "Microfluidic diagnostic technologies for global public health." Nature no. 442 (7101):412-418. DOI: 10.1038/nature05064.

Zhang, Yali, Peng Zuo and Bang-Ce Ye. "A low-cost and simple paper-based microfluidic device for simultaneous multiplex determination of different Types of chemical contaminants in food." Biosensors and Bioelectronics. DOI: 10.1016/j.bios.2014.12.042.

\section{Acknowledgments}

Work supported by project PTDC/SAU-ENB/116929/2010, EXPL/EMS-SIS/2215/2013 and UID/EQU/50020/2013, co-financed by FEDER through COMPETE, QREN and ON2, and by FCT - Fundação para a Ciência e a Tecnologia. R.O.R acknowledges the PhD scholarship SFRH/BD/97658/2013 granted by FCT. A.M.T.S acknowledges the FCT Investigator 2013 Programme (IF/01501/2013), with financing from the European Social Fund and the Human Potential Operational Programme. 